\title{
Efecto de las prácticas durante el manejo pre-faena sobre el rendimiento de la carne de bovinos
}

\author{
Munilla, M.E.'; Vittone, J.S. ; Lado, M. ${ }^{1}$; Romera, S.A. ${ }^{2}$; Teira, G.A. ${ }^{3}$ \\ ${ }^{1}$ EEA INTA Entre Ríos, Argentina. ${ }^{2}$ CNIA INTA Castelar, Buenos Aires. \\ ${ }^{3}$ Facultad Ciencias Alimentación, Univ.Entre Ríos, Argentina. \\ E-mail: munilla.maria $a$ inta.gob.ar
}

\begin{abstract}
Resumen
Munilla, M.E.; Vittone, J.S.; Lado, M.; Romera, S.A.; Teira, G.A.: Efecto de las prácticas durante el manejo pre-faena sobre el rendimiento de la carne de bovinos. Rev. Vet. 32: 1, 48-53, 2021. La calidad de la carne comienza a definirse desde la etapa de producción primaria. Los sistemas intensivos que se orientan a maximizar la producción deben garantizar el bienestar animal y la calidad de la carne. El confort de los animales, la alimentación y la genética contribuyen a la obtención de una res bien conformada. Por el contrario, los eventos de estrés prolongado repercuten negativamente sobre las características y la conservación de los productos cárnicos. Actualmente, los sistemas productivos y el manejo que se brinda a los animales durante la crianza, transporte y manejo pre faena son deficientes para alcanzar el potencial productivo y la calidad requerida por los consumidores. Por lo expuesto, es importante abordar los factores limitantes en la producción y vertirlos en la cadena productiva. El objetivo de la presente revisión es abordar los efectos de las buenas prácticas ganaderas y el manejo previo a la faena sobre el rendimiento de res y la calidad de la carne.
\end{abstract}

Palabras clave: carne vacuna, manejo pre-faena, bienestar animal.

\begin{abstract}
Munilla, M.E.; Vittone, J.S.; Lado, M.; Romera, S.A.; Teira, G.A.: Effect of practices during pre-slaughter management on meat quality of cattle. Rev. Vet. 32: 1, 48-53, 2021. Meat quality begins to be defined since the farm period. Intensive production systems that aim to maximize production must preserve animal welfare and harmlessness meat for consumer safety. Comfort, feeding and genetics contribute to obtaining a well finishing animal. While long-term stress affects the characteristics and preservation of meat products. Currently, the production systems and the management that are provided to the animals during the breeding, transport and pre-slaughter management are deficient to reach the productive potential and the quality required by the consumers. For these reasons, it is important to address the limiting factors in production and pour them into the production chain. The objective of this review is to report the effects of good livestock practices and pre-slaughter management on beef performance and meat quality.
\end{abstract}

Key words: bovine meat, handling pre-task, animal welfare.

\section{Situación actual de la cadena productiva de carne vacuna y perspectivas futuras}

El rodeo nacional de Argentina está integrado por 54 millones de bovinos distribuidos principalmente en el centro y norte del país. De 13,4 millones de cabezas que se faenan anualmente, Buenos Aires participa con el 53,5\%, Santa Fe el 19,8\%, Córdoba el 8,2\%, La Pampa el 3,9\%, Entre Ríos el 3,6\% y el resto de las provincias el $11 \%$.

Las 5 provincias mencionadas integran la Región Pampeana, donde también están dadas las condiciones medioambientales para lograr el $83 \%$ de la producción de soja, trigo, maíz, girasol y sorgo ${ }^{19,38}$.
En los últimos años, el avance de la frontera agrícola en la región Pampeana y el Litoral argentino provocaron la reducción de la superficie asignada a la ganadería. La pérdida de rentabilidad con respecto a la agricultura, la marginalidad de la producción y la necesidad de incrementar la producción de carne para abastecer la creciente demanda internacional, orientaron la producción a modelos intensivos para producir carne. La intensificación está vinculada esencialmente con el uso eficiente de la superficie y con el uso de alimentos que concentran más nutrientes.

De esta manera, se reducen significativamente el espacio y el tiempo del ciclo productivo. El objetivo es mantener o incrementar el stock ganadero en un país que se ha reconvertido en un gran productor de cereales y oleaginosas para el mundo. 
Otro objetivo es lograr una homogeneidad de producto acorde a las exigencias de los consumidores ${ }^{2}$. $\mathrm{Al}$ mismo tiempo, han ocurrido cambios estructurales en la industria frigorífica, principalmente referidos al proceso de exportación que afecta las condiciones de compra y venta de hacienda para faena en el mercado local ${ }^{34}$

Argentina cuenta con 495 frigoríficos distribuidos en la mayoría de las provincias. La mayor concentración se ubica en la región Pampeana y el NEA (especialmente Buenos Aires, Chaco, Entre Ríos, Santa Fe y Córdoba). Esta distribución sigue el patrón que evidencian las actividades ganaderas y se encuentra ligada a la ubicación de los principales mercados de consumo ${ }^{5}$.

Varios frigoríficos se integran para asegurarse disponibilidad continua durante todo el año, evitando picos de precios y disminuyendo costos de transacción ${ }^{21}$. De la producción total, el $43 \%$ de los animales faenados provienen de establecimientos de engorde a corral, los cuales se ubican en su mayoría en la región. En el feedlot $^{31}$ o engorde a corral se prevee el confinamiento y el uso de dietas con alta concentración de energía y digestibilidad con el objetivo de acortar los plazos respecto de planteos extensivos.

Esta condición es valorada por los principales centros de distribución ya que permite garantizar las entregas en tiempo y forma durante todo el año, factor fundamental para la logística de abastecimiento. En estos sistemas de producción de carne, los animales dependen por completo del hombre para satisfacer sus necesidades diarias básicas tales como alimento, agua, refugio y salud ${ }^{32}$.

\section{Relevancia del bienestar animal en la cadena productiva}

El bienestar animal se refiere al estado de armonía del animal con su entorno, considerando su confort, alojamiento, nutrición, prevención de enfermedades, cuidado responsable, manejo y sacrificio humanitario. Por ello es el factor más importante para que los animales alcancen el mejor estado de salud posible y se logre en forma racional un máximo beneficio económico ${ }^{13}$. Los momentos críticos a tener en cuenta son la utilización del animal a campo, su traslado al frigorífico y su manejo previo a la faena.

El bienestar animal se ha integrado a la agenda política en varios países en respuesta a las exigencias de la sociedad. Por ejemplo, en la Comunidad Europea cuentan con un protocolo para el bienestar animal, el cual se incluye dentro del Tratado de Ámsterdam de 1977, que constituye una gran contribución y obliga a las instituciones y estados miembros de la comunidad europea a incorporar el bienestar animal en la producción, transporte, investigación y políticas de mercado ${ }^{9}$.

En ocasiones, el aspecto que más se asocia con la adopción de principios y regulaciones sobre bienestar animal para los animales de granja, es la exportación. Los países que exportan a los mercados más exigentes como la Unión Europea, han adecuado sus regulaciones para dar respuesta a las exigencias de este mercado y desarrollan activamente nuevas iniciativas para abordar el tema.

Esta tendencia abarca obligatoriamente los aspectos de sacrificio y transporte, mientras que los de producción son más bien voluntarios. Por otra parte, están los países que aspiran a ser exportadores y que reconocen la importancia del bienestar animal, ya sea para incorporarlo o para mejorarlo ${ }^{35}$

En Argentina, la preocupación se manifiesta principalmente por exigencia de clientes internacionales, en especial la Unión Europea, que poseen interés tanto en la calidad del producto final como en la característica de los procesos de producción. Pero en el fondo, se considera que este tema afecta a todos los eslabones de la cadena cárnica argentina (criadores, feedloters, transportistas, frigoríficos y comerciantes).

Prestándole atención al bienestar de los animales, se obtienen más y mejores carnes a menor costo. Lejos de constituir una barrera arancelaria o un costo adicional, el cuidado del bienestar animal es una ventaja competitiva para nuestra producción de carne y un significativo ahorro de costos de producción ${ }^{14}$.

La investigación sobre calidad de la canal y de la carne, en la mayoría de los casos se ha hecho separando la producción primaria del producto final. Por una parte, se evalúa el desempeño productivo del ganado (ganancia de peso, consumo de alimento, eficiencia alimentaria, etc) y por otra se evalúa la calidad de la carne, lo cual no permite establecer una relación directa de causa-efecto. Por ejemplo, no es posible determinar qué repercusión tendría utilizar una estrategia particular de producción sobre el comportamiento productivo y la calidad e inocuidad de la carne ${ }^{9}$.

El desarrollo de herramientas y tecnologías de procesos que mejoren el confort de los animales durante la etapa de producción primaria, son necesarios para mejorar la obtención de carne vacuna y facilitar el acceso a mercados internacionales. El respaldo que brinda la disciplina científica también contribuye a su implementación a escala comercial ${ }^{9}$.

\section{Efectos de buenas prácticas durante la crianza, transporte y manejo pre-faena}

Un ambiente satisfactorio para los bovinos brinda condiciones térmicas y físicas confortables que se manifiestan a través de la salud y el comportamiento social adecuado. Los ambientes que no concuerdan con estas características favorecen la aparición de eventos de estrés ${ }^{3}$. El estrés es un indicador de la pérdida de confort animal y es definido como la acción de estímulos provocados por el entorno sobre los sistemas nervioso, endocrino, circulatorio y digestivo de un animal, produciendo cambios en su funcionalidad ${ }^{7}$.

De acuerdo a su duración y frente a los estímulos externos, se manifiesta una combinación de cuatro respuestas de defensa biológica: comportamiento, sistema 
nervioso autónomo, inmunidad y aparato neuroendocrino. Pese a que los cuatro sistemas están disponibles para que el animal responda a un factor estresante, no todos son utilizados de forma simultánea. La homeostasis se mantiene cuando los dos primeros mecanismos están involucrados, pero cuando los cuatro mecanismos de defensa han sido implicados, algunas de las funciones biológicas pueden verse afectadas negativamente y los animales estarán en peligro ${ }^{42}$.

Las condiciones de estrés durante la etapa de producción primaria pueden provocar disminución del consumo, menores ganancias de peso y mayor incidencia de enfermedades que contribuyen a que los animales sean menos eficientes y deban mantenerse por más tiempo hasta alcanzar la terminación deseada para ser enviados a faena. El engorde a corral (feedlot $)^{4}$ ofrece condiciones que contribuyen a la aparición de factores estresantes, principalmente el confinamiento y la presencia de barro en los corrales ${ }^{12}$. El barro es el peor enemigo del feedlot ${ }^{6,10,23}$

Los establecimientos ganaderos con engorde a corral situados en regiones con precipitaciones de más de $500 \mathrm{~mm}$ anuales, presentan dificultad para mantener los corrales secos y se enfrentan a la formación de barro ${ }^{16}$. En las regiones de Argentina con menos de $600 \mathrm{~mm}$ anuales, la evaporación es altamente eficiente para reducir los volúmenes de líquidos recogidos en el feedlot. En zonas con 600-1.200 mm anuales, la instalación se torna más compleja y en regiones con más de $1.200 \mathrm{~mm}$ anuales no sería aconsejable instalar feedlots ${ }^{33}$.

De acuerdo a estos parámetros, la región pampeana y gran parte del litoral argentino poseen una cantidad excesiva de precipitaciones para instalar un feedlot sin la infraestructura necesaria, y aun así el manejo de efluentes y la formación de barro son una limitante operativa. En un feedlot de 1.000 animales se producen diariamente entre 3 y 5 tn de estiércol y el ritmo de producción es superior al secado ${ }^{26}$. Así, el manejo de los efluentes generados se vuelve incontrolable.

De todas estas externalidades negativas de los encierres, la producción de olores desagradables y la acumulación de barro en los corrales son las rechazadas por la sociedad en su conjunto. En sistemas de engorde a corral, la asignación de superficie es de 10 a $20 \mathrm{~m}^{2} /$ animal. Una de las alternativas más accesibles y sencillas para incorporar y disminuir los efectos negativos del confinamiento, es brindar más superficie para los animales.

Expertos norteamericanos reportaron que en regiones con $720 \mathrm{~mm}$ de precipitaciones anuales, la asignación de $46 \mathrm{~m}^{2}$ / animal son suficientes para mantener el corral seco ${ }^{25}$. Además, si se duplica la superficie asignada, es posible reducir la formación de barro y las consecuencias negativas sobre la producción 18,24

Los efectos del estrés ante-mortem relacionados con el transporte y el manejo pre faena han sido estudiados en profundidad, especialmente en países donde el transporte desde el lugar de origen hasta el sitio de faena implica varias horas de ayuno y se dan las con- diciones para que los animales manifiesten un estrés agudo, repercutiendo sobre la calidad de la carne ${ }^{40}$. Además de ello, los antecedentes de los animales (genética y edad), el ambiente y la alimentación deben ser integrados al estudio de los efectos del manejo sobre la producción y la calidad de la carne.

Para producir carne con atributos de calidad, se deben comprender los factores que afectan dichos parámetros. La terneza es afectada por la interacción de la genética y el ambiente. Esta característica de la carne se determina a partir de la contribución del tejido conectivo, la longitud de los sarcómeros, la tasa de proteólisis durante el envejecimiento de los animales, la presencia de grasa intramuscular y el metabolismo energético post-mortem. El estrés también posee injerencia sobre la terneza.

El estrés provoca la liberación de adrenalina como respuesta natural que prepara al cuerpo para la lucha o huida ante una amenaza. Fisiológicamente, provoca la tensión de los músculos y disminuye su $\mathrm{pH}$. Cuando el estrés del ganado es a corto plazo, los cambios temporales en la química de la carne hacen que ella se endurezca y pierda sabor. Pero si el estrés persiste durante un período prolongado de tiempo, la persistente acidez afecta la composición de la carne. El estrés a largo plazo también afecta los órganos vitales del bovino, que deben procesar y expulsar los compuestos generados por el estrés. Sin embargo, puede ser difícil de reconocer dado que la intensidad del estrés no es constante y no siempre se encuentra asociado a patologías sintomáticas ${ }^{29}$.

El estrés de largo plazo también favorece la aparición de carnes oscuras. El glucógeno se libera en el músculo y se convierte en ácido láctico causando una alteración en el descenso de $\mathrm{pH}$, dando al momento de la faena un tejido magro de color oscuro ${ }^{37}$. Se ha reportado que la mezcla de animales de diferentes orígenes en el feedlot y las fluctuaciones del clima, entre otros factores, predisponen la aparición de la carne DFD (oscura, firme y seca) ${ }^{27}$.

Los productos cárnicos ideales para la exportación y consumo interno son aquellos que cumplen una serie de requisitos tales como seguridad alimentaria, calidad sensorial acorde a las exigencias del consumidor, bienestar animal y funcionalidad. En este contexto, el desarrollo e implementación de estrategias de producción de carne bovina resultan relevantes para alcanzar los estándares de calidad exigidos por el mercado actual. Está fuera de discusión que los vacunos engordados con dietas energéticas exhiben una marcada uniformidad de terminación y su carne presenta características constantes a lo largo del año. Ambas particularidades son demandadas por los frigoríficos y por los consumidores ${ }^{11}$.

La composición de la carne se establece completamente durante toda la vida del animal, mientras que su calidad se ve fuertemente afectada por factores antemortem y post-mortem ${ }^{30}$. Especialistas pusieron de manifiesto la importancia de los factores intrínsecos del animal (raza, genotipo, sexo, edad, peso) y los fac- 
tores productivos y medioambientales, sobre la calidad de la canal (peso, conformación, engrasamiento) ${ }^{36}$.

Las características fundamentales para obtener una res de alto rendimiento industrial son: una óptima conformación y una justa terminación. Ahora bien, una buena conformación puede ser desvalorizada por una mala terminación, y a la vez una buena terminación no corrige una mala conformación. La conformación se refiere a la proporción y distribución del tejido muscular en las zonas de mayor valor comercial, por ejemplo en el cuarto trasero.

Dicho valor está sujeto a la correcta distribución de las masas musculares y la grasa adyacente, que deberá desarrollarse de forma uniforme. La falta de uniformidad indica alteraciones de orden nutricional en la crianza y el engorde. También tiene importancia el color de la grasa, que debe ser blanco cremoso o nacarado, dado que es un indicador de precocidad y buena alimentación.

La terminación, o cantidad de grasa que recubre a la res, debe guardar relación con las masas musculares pero varía de acuerdo con el mercado para el cual está destinado. La res ideal para cualquier mercado tiene que presentar la máxima cantidad de músculo en relación a su esqueleto y la cantidad de grasa exigida por el mercado a la que va destinada, pero no más, ya que en exceso disminuye el valor de la res ${ }^{12}$.

El factor que más afecta el rendimiento es el tipo de dieta. Dietas con una mínima cantidad de fibra presentan mayor concentración energética y se ha demostrado que eleva el rendimiento ${ }^{8,12,17}$. La grasa es responsable, en parte, del sabor de la carne, y resulta necesaria para la conservación de la carcasa. Puesto que hay que recortar el exceso de grasa en la carne, se la considera un producto desperdiciado y, en consecuencia, los consumidores suelen rechazar el exceso de grasa, independientemente de su contribución a la calidad del producto ${ }^{22}$.

La terneza es uno de los aspectos más importantes para determinar la calidad de la carne. Su grado de variabilidad es el principal factor de insatisfacción por parte de los consumidores. Las diferencias pueden deberse a la edad, alimentación, genética y composición de la carcasa. Entre los factores post-mortem que la afectan se encuentran la estimulación eléctrica, el enfriamiento, la conservación y el métodos de tiernización. La composición de la carcasa y la deposición de grasa, que dependen de factores ante-mortem, juegan un rol importante sobre el marmolizado de la carne y la terneza. Para mantener elevados grados de terneza, es necesario implementar buenas prácticas desde la etapa de producción primaria ${ }^{41}$

La reducción del estrés y no limitar la alimentación con recursos de buena calidad contribuyen a la continuidad de deposición de grasa intramuscular ${ }^{1}$. La velocidad e intensidad con que el $\mathrm{pH}$ desciende luego de la faena, está principalmente determinada por la cantidad de ácido láctico que pueda acumularse a partir de la utilización anaeróbica del glucógeno muscular. Las reservas de glucógeno son -por lo tanto- de suma im- portancia para determinar la calidad de la carne. Tal cantidad está relacionada con el tipo de alimentación y con el nivel de estrés antes de la faena.

Dietas con altas concentraciones de energía tales como las of recidas en el engorde intensivo, permiten incrementar las reservas musculares de glucógeno, debido a una mayor disponibilidad de propionato para la gluconeogénesis a nivel muscular. Por lo tanto, altas reservas de glucógeno generadas por la participación de grano en la dieta y la minimización del estrés de los animales antes de la faena, permitirán disponer de altas reservas de glucógeno muscular susceptibles de ser transformado en ácido láctico. Esto permitirá que el $\mathrm{pH}$ descienda rápidamente hasta niveles compatibles con una adecuada conservación de la carne ${ }^{15,28}$.

La calidad de la carne está determinada por su composición química y por sus características organolépticas, tales como terneza, color, olor, sabor y jugosidad. El sistema de producción, el tipo de animal, el plano nutricional ofrecido, y el manejo pre y post faena, pueden modificar considerablemente estas características ${ }^{15}$. El ritmo de ganancia de peso de los animales puede contribuir a la mejoría de la terneza: altas ganancias de peso producen una mayor velocidad de recambio proteico a nivel muscular.

Elevadas ganancias determinan mayor cantidad y actividad de las enzimas responsables de la degradación de las fibras musculares y -por lo tanto-se logra mayor terneza. A su vez, el colágeno que rodea a las fibras musculares condiciona en gran medida la terneza de la carne debido a su alta resistencia. Frente a altas ganancias de peso, el mismo presenta una mayor solubilidad, producto del mayor recambio proteico, lo cual contribuye a una mayor terneza ${ }^{15}$.

En la actualidad los mercados nacionales e internacionales demandan que los alimentos de origen cárnico sean inocuos y seguros para el consumo humano. Los consumidores y los gobiernos están cada vez más atentos a la seguridad alimentaria, entendida como la certeza de que los alimentos son inocuos para la salud humana.

Esto ha llevado a mayores exigencias en torno a las condiciones de higiene en que se elaboran los alimentos, al no uso de hormonas como método para lograr productos de determinadas características, así como al uso de antibióticos y a los componentes del forraje. Estas crecientes exigencias son particularmente severas en los países desarrollados, pero tienden a generalizarse cada vez más ${ }^{20}$.

La carne de bovino forma parte de la dieta integral alimenticia de la humanidad, principalmente por el alto valor nutricional que representa y para producir una carne segura y sana, es necesario modificar e implementar nuevas prácticas de producción pecuaria ${ }^{35}$. Las malas prácticas ganaderas generan un perjuicio económico evitable en el valor del animal y atentan contra la calidad y el rendimiento de la carne de nuestro país. El desarrollo constante de estrategias sobre el bienestar animal basadas en los análisis de riesgos y puntos críticos, 
hará posible la integración paulatina del bienestar animal y las normas relativas a la seguridad alimentaria ${ }^{5}$.

\section{Legislaciones para contribuir al bienestar animal y la calidad de la carne}

La primera ley para la protección de los animales se sancionó en el año 1822 en Inglaterra, la cual posteriormente dio lugar a la fundación Royal Society for the prevention of cruelty to animals. En el año 1954, Argentina promulgó la ley 14.346 que -aún activa- contempla la protección de los animales con relación a los malos tratos y actos crueles por parte de los seres humanos. Más allá de estos antecedentes, la institucionalización de la problemática del bienestar animal tiene fecha conocida de ocurrencia.

El libro Animal Machines, escrito por Ruth Harrison en 1964, abrió las puertas al debate sobre la ética de la producción animal en la agricultura del Reino Unido de Gran Bretaña. El impacto que la publicación generó en la sociedad británica llevó al Parlamento a la creación del Comité Brambell, que en 1965 postuló las 5 libertades mínimas de las que todo animal debería gozar (voltearse, cuidarse corporalmente, levantarse, echarse y estirar los miembros).

Este comité fue el primero en proponer una definición científica de bienestar. Sus miembros comenzaron a tratar la importancia del comportamiento en relación al bienestar animal, destacaron la necesidad de su estudio científico y aceptaron que los animales tenían sentimientos, lo cual iba en contra de la tendencia conductista del momento.

En 1993, el Consejo de Bienestar para los Animales de Granja (Farm Animal Welfare Council) del Reino Unido, formuló las nuevas cinco libertades, aún vigentes, como una forma de mejorar las anteriormente propuestas por el Comité Brambell.

La Asociación Mundial de Veterinarios (AMV) acepta y afirma la primacía de la profesión en el diagnóstico, tratamiento y control de las enfermedades animales, pero también reconoce la responsabilidad sobre todo lo necesario para disminuir el sufrimiento, dolor y angustia, promoviendo el bienestar animal (BA).

El Comité Permanente de la AMV, desde mayo de 1990, adoptó una política sobre el BA con alcance mundial, la que es difundida y promovida a través de los miembros representantes por los continentes y subcontinentes (África, América Latina, Asia, Australasia y Europa) ante el comité respectivo sobre "BA y Etología". A través de las actividades de este comité, en todos los países se trata de estimular la implementación de las politicas sobre bienestar animal aprobadas por la AMV.

\section{Conclusiones}

El rendimiento y la calidad de la carne están determinados por el manejo que reciben los animales durante toda su vida. Las buenas prácticas contribuyen claramente en la mejoría de la calidad de la carne ${ }^{39}$.
Además de las legislaciones vigentes y las posibles 1imitaciones para exportar, es fundamental dar relevancia al cambio de paradigma y comenzar a visualizar a las buenas prácticas durante la crianza, transporte y manejo pre faena de los bovinos, para contribuir a la producción de carne con los atributos de calidad que los consumidores exigen.

\section{REFERENCIAS}

1. AG Info Centre. 2007. Management practices to improve marbling and beef quality. https://wwwl.agric.gov. ab.ca/Sdepartment/deptdocs.nsf/all/faq11416

2. Alassia G, Gatti Z, Stefanazzi D. 2008. Proyecto de inversión de engorde bovino a corral. Facultad de Agronomía, Universidad Nacional La Pampa. http://www.agro. unlpam.edu.ar/licenciatura/diseno/engordebob.pdf

3. Alende M. 2010. Bienestar animal y reducción del estrés en el feedlot. https://inta.gob.ar/sites/default/files/scripttmp-bienestar animal.manejo.racional en feed lot.pdf.

4. Andrews R. 2013. Cattle feedlot: behind the scenes. https:/www.precisionnutrition. com/cattle-feedlot-visit

5. Bergaglio OE. 2013. La práctica de bienestar animal: una ventaja competitiva para el ganado y la carne en la $\mathrm{Ar}$ gentina. Apuntes agroeconómicos, Facultad de Ciencias Agrarias, UBA. https://www.agro.uba.ar/apuntes/no_8/ bienestar.htm

6. Boyles S. 2001. Feedlot mud management. Ohio University State, Ohio Beef Cattle Letter. http://u.osu.edu/ beef/2001/06/13/feedlot-mud-management

7. Broom DM. 2005. The effects of land transport on animal welfare. Rev Sci Tech off Int Epiz 24: 683-691.

8. Bucy LV, Bennion LL. 1962. Concentrate-roughage levels in diets of fattening steers. J Anim Sci 21: 3, 666.

9. Carrillo DS, Calvo CC, Lozano L, Huerta BM. 2015. Bienestar animal, inocuidad y calidad de productos pecuarios. Universidad de Chapingo. https:/chapingo.mx/produccionanimal/images/stories/documentos/Lineas_de_investigacion/Bienestar_animal_inocuidad_calidad.pdf

10. Colombatto D. 2013. El feedlot es una herramienta y no un fin en sí mismo. https:/www.rionegro.com.ar/pulso/ colombatto-el-feedlot-es-una-herramienta-y-no-un-finen-si-mismo-IBRN 7859723

11. Ferrari O. 2013. Ganadería eficiente: claves para el manejo productivo. Ministerio de Agricultura, Ganadería y Pesca de la Nación. ISBN: 978-987-1873-21, 233 p.

12. Ferrari O, Speroni NA. 2008. Feedlot actual. Ministerio de Agricultura, Ganadería y Pesca de la Nación. ISBN: 978-987-23200-1-0, $430 \mathrm{p}$.

13. Gil F. 2005. Bienestar animal y su impacto económico. Asociación Argentina de Criadores de Hereford, Buenos Aires, Vol. 637, pág. 66-79.

14. Giménez ZM. 2006. El bienestar animal y la calidad de la carne: buenas prácticas de manejo de ganado. IPCVA, Cuad.Téc. ${ }^{\circ} 1$,http://www.ipeva.com.ar/files/ctl.pdf

15. Grigera JM, Rearte D, Santini FJ. 2003. Algunos aspectos sobre la calidad de las carnes bovinas asociadas a los sistemas de producción. $1^{\circ}$ Jorn Act Ganadera, Balcarce. http://www.ipcva.com.ar/vertext.php?id=148 
16. Grandin T. 2016. Evaluation of the welfare of cattle housed in outdoor feedlot pens. Veterinary and Animal Science 1/2: 23-28.

17. Guenther JJ, Bushman DH, Pope LS, Morrison LD. 1965. Growth and developement of the major carcass tissues in beef calves from weaning to slaughter weight, with reference to the effect of plane of nutrition. Journal of Animal Science 24: 1184-1191.

18. Holland B. 2012. Preventing muddy conditions in feedlot pens. SDSU Ext.Beef Feedl.http://igrow.org/livestock/ beef/preventing-muddy-conditions-in-feedlot-pen.

19. IPCVA: Instit. Prom. Carne Vacuna. 2018. Faena y producción de carne vacuna. http://www.ipcva.com.ar/documentos /1965_1548258434_informein4trimestre.pdf

20. Laens S, Paolino C. 2004. Estudio de competitividad de cadenas agroindustriales. Centro Investig. Económ. http://www.funcex.org.br/material/redemercosul.17pdf.

21. Lamas MF, Ponti D. 2014. Logística integral, etapa de producción primaria: de la cabaña al frigorifico. Serie $\mathrm{N}^{\circ}$ 3. Ministerio de Agricultura, Ganadería y Pesca. https:// www.agroindustria.gob.ar/sitio/areas/bovinos/informacion

22. Lobato L. 2008. Terneza y calidad de la carne bovina. News Agro Argentina. http://www.agrositio.com/vertext/ vertext.php?id $=89594 \& \mathrm{se}^{=}$

23. Machado MP. 2012. Etología bovina. 73 p. http://www. produccion-animal.com.ar/etologia_y_bienestar/etologia bovinos/22-TESIS_etologia.pdf

24. Mader T. 2011. Mud effects on feedlot cattle. Nebraska Beef Cattle Report. https:/beef.unl.edu/c9405542-1c414b9c-al43-f192ele72917.pdf

25. Mader T, Griffin D. 2015. Management of cattle exposed to adverse environmental conditions. Vet.Clin.North.Am. https://www.ncbi.nlm.nih.gov/pubmed/26139190

26. Miller M. 1998. Factors contributing to the incidence of the dark cutting condition in beef carcasses. https://www. beefresearch.org/CMDocs/BeefResearch/PE

27. Monteiro M, Peluffo M. 2002. Terneza: una caracteristica a tener en cuenta. www.produccionbovina.com/informacion_tecnica/carne_y_subproductos $/ 00$

28. National Research Council (NRC). 1992. Recognition and alleviation of pain and distress in laboratory animals. https:/Www.nap.edu/read/1542/chapter/1\#ii

29. Onega ME. 2003. Evaluación de la calidad de carnes frescas: aplicación de técnicas analíticas, instrumentales y sensoriales. http://biblioteca.ucm.es/tesis/vet/ucm
30. Organización Mundial de Sanidad Animal (OIE). 2013. Código Sanitario para los animales terrestres. Vol. $\mathrm{N}^{\circ} 1$, Ed. N ${ }^{\circ} 22$, ISBN 978-92-9044-914-0.

31. Pordomingo AJ. 2003. Gestión ambiental en el feedlot, guía de buenas prácticas. https://inta.gob.ar/sites/default/ files/script-tmp-gestin-ambiental.pdf

32. Robert S, Santangelo F, Albornoz I, Dana G. 2010. Estructura del feedlot en Argentina. Nivel de asociación entre la producción bovina a corral y los titulares de faena. http://www.ipcva.com.ar

33. Rojas H, Stuardo L, Benavides D. 2005. Politicas y prácticas de bienestar animal en los paises de América: estudio preliminar. Rev Sci Tech 24: 549-565.

34. Romero MH, Uribe LF, Sánchez JA. 2011. Biomarcadores de estrés como indicadores de bienestar animal en ganado de carne. Biosalud 10: 1, ISSN 1657-9550.

35. Sañudo C. 1993. Calidad organoléptica de la carne. Tecnología y Calidad de los productos cárnicos. Curso 8-12 junio, Pamplona.

36. Scanga JA, Belk KE, Tatum JD, Grandin T, Smith GC. 1998. Factors contributing to the incidence of dark cutting beef. J Anim Sci 76: 2040-2047.

37. SENASA (Servicio Nacional de Sanidad y Calidad Agroalimentaria. 2018. Distribución de existencias bovinas por provincias. https://www.argentina.gob.ar/files/2 distribuciondeexistenciasbovinasporprovinciamarzo2018xlsx

38. SENASA (Servicio Nacional de Sanidad y Calidad Agroalimentaria. 2013. Establecimientos de engorde a corral 2008-2013. https://www.argentina.gob.ar/sites /default/files/11.1_2013-2008_0.pdf

39. Špehar M, Vincek D, Žgur S. 2008. Beef quality: factors affecting tenderness and marbling. Department of Animal Science 62: 463-478.

40. Trevisi E, Bertoni G. 2009. Some physiological and biochemical methods for acute and chronic stress evaluation in dairy cows. J Anim Sci Supl.1: 265-286.

41. Voisinet BD, Grandin T, O'Connor SF, Tatum JD, Deesing MJ. 1997. Bos indicus - Cross feedlot cattle with excitable temperaments have tougher meat and a higher incidence of borderline dark cutters. Meat Science 46: 367377.

42. Warner RD, Greenwood PL, Pethick DW, Ferguson DM. 2010. Genetic and environmental effects on meat quality. Meat Science 86: 171-183. https:/www.researchgate.net/publication/44686385_Genetic_and_environmental_effects_on_meat_quality. 